\title{
Fhit, Mlh1, P53 and phenotypic expression in the early stage of colorectal neoplasms
}

\author{
AKIKO YASUGI, KAZUO YASHIMA, AKIHITO HARA, MASAHARU KODA, KOICHIRO KAWAGUCHI, \\ KENICHI HARADA, HIRONOBU ANDACHI and YOSHIKAZU MURAWAKI
}

Division of Medicine and Clinical Science, Faculty of Medicine, Tottori University, Yonago 683-8504, Japan

Received May 24, 2007; Accepted August 6, 2007

\begin{abstract}
There are two different pathways for the development of colorectal carcinoma (CRC), adenomacarcinoma sequence (ACS) and de novo (DN) carcinogenesis. To clarify the molecular and clinicopathological characteristics in colorectal carcinogenesis, we examined endoscopically resected specimens of 30 adenomas, 30 carcinoma in adenomas (CIAs), and 18 early pure colorectal carcinomas without any adenoma component (EPCs, so called DN carcinoma) and compared the expression of Fhit, Mlh1, Msh2, P53 and cellular phenotype (HGM, MUC2 and CD10). Markedly reduced or absent Fhit expression was noted in $8(44 \%)$ of $18 \mathrm{EPCs}$, but none of the adenomas or CIAs $(p<0.0001)$. Six $(33 \%)$ of 18 EPCs showed loss of Mlh1 expression, but rarely in adenomas and CIAs ( $\mathrm{p}=0.008)$. This altered Fhit expression was significantly higher in submucosal invasive cancers $(\mathrm{p}=0.001)$, lymphatic or venous invasive cancers $(\mathrm{p}=0.0018)$, and tumors with altered expression of Mlh1 ( $\mathrm{p}=0.01)$. The incidence of P53 overexpression was significantly higher in EPCs (39\%) and CIAs $(27 \%)$ than in adenomas $(3.3 \%)(\mathrm{p}<0.05)$. There were significant differences in phenotypic expression between the adenomatous and carcinomatous areas. Moreover, in CIAs and EPCs, the rate of P53 overexpression was significantly higher in the CD10-positive cases (53\%) than CD10-negative cases $(19 \%)(\mathrm{p}=0.04)$. The present findings suggested that aberrant Fhit and Mlh1 expression could be related to DN carcinogenesis and that P53 overexpression and changes in phenotypic expression could contribute to the malignant transformation of colorectal precursor lesions.
\end{abstract}

Correspondence to: Dr Kazuo Yashima, Division of Medicine and Clinical Science, Faculty of Medicine, Tottori University, Yonago 683-8504, Japan

E-mail: yashima@grape.med.tottori-u.ac.jp

Abbreviations: ACS, adenoma-carcinoma sequence; $\mathrm{DN}$, de novo; CIA, carcinoma in adenoma; EPC, early pure colorectal carcinoma; MMR, mismatch repair; CRC, colorectal cancer

Key words: Fhit, Mlh1,p53, mucin phenotype, colorectal cancer

\section{Introduction}

On the basis of clinicopathological features, two different pathways for colorectal carcinogenesis have been proposed. The adenoma-carcinoma sequence (ACS) proposes that the carcinoma arises from a pre-existing adenoma, whereas the second pathway is de novo (DN) development of the carcinoma (1-3). DN carcinoma is thought to develop directly from non-neoplastic colorectal epithelia without adenomatous precursors. Previous reports emphasized that these lesions appeared to be more aggressive than the conventional ACS type carcinomas, since they had a tendency to more deeply invade the colonic wall at a smaller size (3-5).

A candidate tumor suppressor gene, Fragile Histidine Triad (FHIT), was identified at chromosome 3p14.2 spanning the FRA3B common fragile site. Abnormalities and reduced expression of the FHIT gene have been found in a variety of human cancer cell lines and primary tumors $(3,6)$. It was also suggested that Fhit plays a role in the development and progression of colorectal carcinoma (CRC) (7-10). Recent studies have revealed that reduced Fhit protein expression is associated with a deficiency of mismatch repair (MMR) in human advanced CRCs (11-13). However, there are few reports in relation to early colorectal carcinogenesis.

Gastric and intestinal phenotypic expression is important for the histogenesis of cancers of the digestive tract. We and others have previously demonstrated a relationship between clinicopathological findings and phenotypes using gastric and intestinal phenotypic markers such as HGM, MUC2 and CD10 in stomach cancers (14-17). Changes were also seen in intestinal metaplasia, a putative precancerous lesion for stomach cancers (18-20). Several reports have pointed to changes of phenotypic expression in colorectal carcinogenesis and thus it is of interest to analyze links between phenotypic expression and pathways of colorectal carcinogenesis, especially from adenomas to early CRCs, and with progression (21-25).

In the present study, Fhit, Mlh1, Msh2, P53 and phenotypic expression were analyzed in early colorectal neoplasms. The results indicate that aberrant Fhit and Mlh1 expression could be related to DN carcinogenesis and that P53 and phenotypic expression could contribute to the malignant transformation of colorectal precursor lesions. 


\section{Materials and methods}

Patient samples. Tumor specimens were obtained from 78 patients (46 males and 32 females), who had undergone endoscopic resection at Tottori University Hospital. There were 3 groups $/ 30$ adenomas, 30 carcinoma in adenomas (CIAs), and 18 early pure carcinomas without any adenoma component (EPCs). Adenomas and CIAs measuring more than $10 \mathrm{~mm}$ were selected. All histologic types of CIAs and EPCs were well differentiated adenocarcinomas. Moreover, these neoplasms were confined to the mucosa or submucosa. We speculated that EPC groups should contain DN carcinomas. Histological evaluations were made according to the classification established by the Japanese General Rules for Clinical and Pathological Studies on Cancer of the Colon, Rectum and Anus (26). In this study, adenoma samples corresponded to low or high grade adenoma/dysplasia, and CIA and EPC samples confined to the mucosa and those confined to the submucosa corresponded to non-invasive carcinoma or intramucosal carcinoma and submucosal carcinoma in the Vienna classification system, respectively. The macroscopic features were use to divide tumors into two major types: polypoid, and flat or depressed. A flat and depressed tumor was defined as an endoscopically visible flat and/or depressed mucosal lesion with a height that was less than half the diameter of the lesion (27). All other tumorous lesions in the colon were termed polypoids. The clinical characteristics of the patients are listed in Table I. Pathological diagnoses were verified by two experienced pathologists (H.A. and H.I.). All the cases were analyzed anonymously, that is, all the specimens were assigned a new number without any personal information. Institutional Review Board approval was obtained.

Immunohistochemical staining. Paraffin-embedded, $4 \mu \mathrm{m}$ thick sections were immunohistochemically stained with an anti-Fhit rabbit polyclonal antibody (IBL, Gunma, Japan; dilution 1:100), an anti-MSH2 mouse monoclonal antibody (FE11, Oncogene Research Products, Boston, MA, USA; 1:100); anti-MLH1 mouse monoclonal antibody (G168-15, PharMingen, San Diego, CA, USA; 1:50), an anti-P53 mouse monoclonal antibody (DO-7, Dakopatts, Copenhagen, Denmark; 1:50), an anti-human gastric mucin (HGM) mouse monoclonal antibody (45M1, Novocastra Laboratories, Ltd., Newcastle, UK; 1:50), an anti-MUC2 mouse monoclonal antibody (Ccp 58, Novocastra; 1:100) and an anti-CD10 mouse monoclonal antibody (56C6, Novocastra; 1:50) using the avidin-biotin-peroxidase complex technique.

Immunohistochemical staining was performed as described below. In brief, after deparaffinization in xylene and rehydration in ethanol, the sections were immersed in a citrate buffer $(0.01 \mathrm{M}, \mathrm{pH} 6.0)$ and heated in a microwave oven for 20-30 min to retrieve antigens, then incubated with the primary antibody overnight at $4^{\circ} \mathrm{C}$. As a negative control, the primary antibody was replaced with normal serum $\mathrm{IgG}$ in a similar dilution. The detection reaction was carried out with a Vectastain Elite ABC kit (Vector Laboratories, Burlingame, CA, USA). Diaminobenzidine was used as a chromogen, and hematoxylin as a couterstain. The sections were incubated with biotinylated anti-rabbit or mouse $\mathrm{IgG}$ and avidin-biotin-
Table I. Clinicopathological features in early colorectal neoplasms.

\begin{tabular}{lccc}
\hline & $\begin{array}{c}\text { Adenoma } \\
(\mathrm{n}=30)\end{array}$ & $\begin{array}{c}\text { Carcinoma } \\
\text { in adenoma } \\
(\mathrm{n}=30)\end{array}$ & $\begin{array}{c}\text { Early pure } \\
\text { carcinoma } \\
(\mathrm{n}=18)\end{array}$ \\
\hline Age (year \pm SD) & $69.8 \pm 9.3$ & $67.9 \pm 9.5$ & $70.0 \pm 8.2$ \\
Gender (male/female) & $20 / 10$ & $16 / 14$ & $10 / 8$ \\
$\begin{array}{l}\text { Size (mm } \pm \text { SD) } \\
\text { Location }\end{array}$ & $13.7 \pm 5.5$ & $17.7 \pm 8.5$ & $10.3 \pm 3.2$ \\
(right/left) & $16 / 14$ & $9 / 21$ & $5 / 13$ \\
$\begin{array}{l}\text { Macroscopic } \\
\text { classification (flat or } \\
\text { depressed/polypoid) }\end{array}$ & $1 / 29$ & $1 / 29$ & $12 / 6$ \\
$\begin{array}{l}\text { Depth of invasion } \\
\text { (mucosa/submucosa) }\end{array}$ & - & $24 / 6$ & $7 / 11$ \\
$\begin{array}{l}\text { Lymphatic invasion } \\
\begin{array}{l}\text { Venous invasion } \\
\hline\end{array}\end{array}$ & - & 0 & 6 \\
\hline
\end{tabular}

peroxidase and visualized using diaminobenzidine tetrahydrochloride. The protein expression was evaluated by two independent observers (H.A. and K.Y.). The immunohistochemical analysis was performed in a blinded manner with respect to the clinical information.

Assessment of Fhit immunostaining. The expression of Fhit was graded for both the extent and intensity of immunopositivity as described previously (8). The extent of positivity was scored as follows: $0,<5 \% ; 1,5-25 \% ; 2,25-50 \% ; 3,50-$ $75 \%$; and $4,>75 \%$ of the colonic epithelial cells in the respective lesions. The intensity was scored as follows: 0 , negative; $1+$, weak; $2+$, moderate; and $3+$, as strong as normal mucosa. The final score was obtained by multiplying the positivity and intensity scores, producing a range from 0 to 12 . Scores 9-12 were defined as a preserved or strong staining pattern, scores 5-8 were defined as an intermediate staining pattern, and scores 0-4 were defined as a markedly reduced or lost expression. Normal colorectal epithelia adjacent to the tumor cells showed moderate to strong cytoplasmic expression of the Fhit protein from the basal portion to the luminal differentiated cells; these findings served as an internal positive control. Smooth muscle cells and inflammatory mononuclear cells were positive to various intensities and degrees.

Assessment of Mlh1 and Msh2 immunostaining. Normal tissue adjacent to the tumor was used as an internal positive control. The normal staining pattern for both Mlh1 and Msh2 was nuclear. Cases with definite nuclear staining in $>30 \%$ of the tumor cells were categorized as positive, while cases with definite nuclear staining in $<30 \%$ of the tumor cells were categorized as negative (28). In the normal mucosa, staining was detected predominantly in the areas of active proliferation, 
A

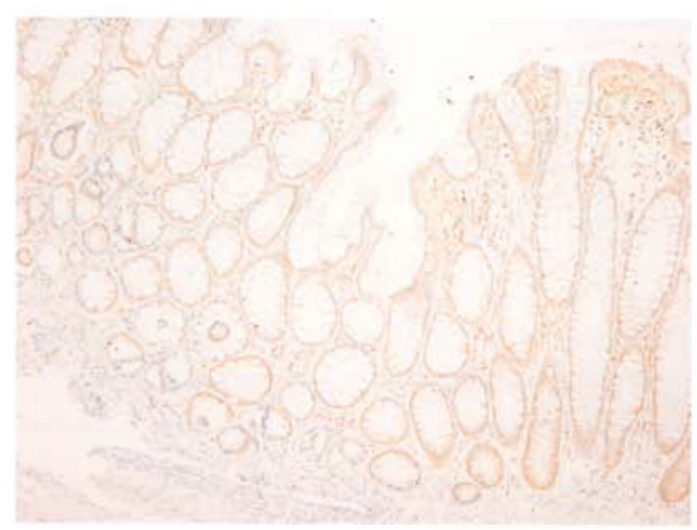

$\mathbf{B}$

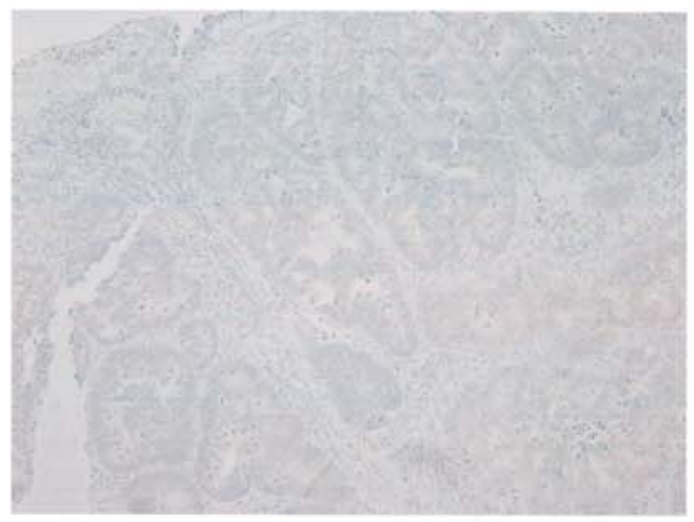

C



D

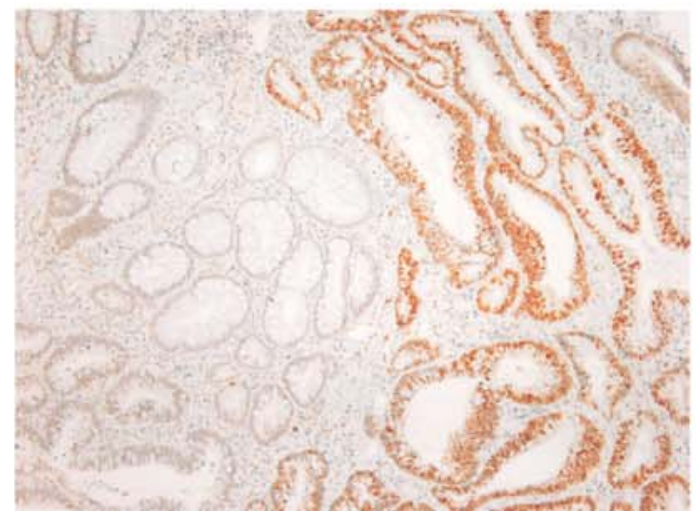

Figure 1. Representative results of Fhit, Mlh1 and P53 immunostaining in normal and neoplastic tissue. (A) Positive Fhit immunostaining of normal colonic epithelium. (B) Negative Fhit immunostaining of an EPC. (C) Negative Mlh1 immunostainig of an EPC and positive Mlh1 immunostaining of a normal colonic epithelium. (D) Positive P53 immunostaining of a carcinomatous area of CIA and negative P53 immunostaining of a normal colonic epithelium. such as the germinal centers of the lymphoid follicles and the lower portions of the normal colonic crypts. Normal stromal cells such as fibroblasts and endothelial cells also showed nuclear positivity for both the proteins.

Assessment of P53 immunostaining. Five representative fields were examined, and a total of 1000 tumor cells (200 for each field) were counted under the microscope with a high power (x200) objective. A distinct nuclear immunoreaction was assessed as positive. In this study, the specimens were regarded as p53-positive when over $25 \%$ of tumor cells showed positive signals.

Assessment of HGM, MUC2 and CD10 immunostaining and classification of the phenotypes. In the normal alimentary tract, HGM staining was seen in the cytoplasm of the gastric foveolar epithelium and mucous neck cells. MUC2 staining was seen in the cytoplasm around the nuclei of goblet cells. CD10 staining was seen along the brush border of the luminal surface of the epithelium. Although CD10 can also be expressed in the apical portion of the cytoplasm in normal colonic mucosa, only the expression of CD10 on the brush border was studied. The results of staining were categorized as either positive or negative. Staining of $>10 \%$ of the adenoma and carcinoma cells was classified as positive expression and that of $<10 \%$, as negative expression.

Statistical analysis. The statistical analysis was performed with the Fisher's exact test, $\chi^{2}$ test with Yates' correction, Kruskal-Wallis or Mann-Whitney U test. P-values $<0.05$ were considered statistically significant.

\section{Results}

Clinicopathological features. The clinicopathological features of the subjects are summarized in Table I. The size of CIAs were larger than that of adenomas and EPCs $(\mathrm{p}<0.04)$. There was no significant difference between adenomas, CIAs, and EPCs in any of the factors other than size. Twelve of 18 EPCs were flat elevated or depressed, whereas 29 of 30 adenomas and 29 of 30 CIAs were polypoid features. Six of 30 CIAs and 11 of 18 EPCs invaded the submucosa. None of the CIAs showed lymphatic and venous invasion, 6 of EPCs showed lymphatic invasion, and 4 of EPCs showed venous invasion.

Fhit, Mlh1, Msh2 and P53 expression in early colorectal neoplasms. Reduced or absent Fhit expression was noted in $8(44 \%)$ of EPCs, but none of the adenomas or CIAs $(\mathrm{p}<0.0001)$. Six (33\%) EPCs showed loss of Mlh1 expression, compared to only $1(3 \%)$ adenoma and $1(3 \%)$ CIA ( $\mathrm{p}=0.008)$. Loss of Msh2 expression was detected in one of the 18 EPCs, but none of the adenomas or CIAs. The incidence of P53 overexpression was significantly higher in EPCs (39\%) and CIAs $(27 \%)$ than in adenomas $(3 \%)(\mathrm{p}<0.05)$. Among the tumors with reduced or absent Fhit expression, 4 (50\%) of 8 showed loss of Mlh1 and/or Msh2 expression, compared to just $3(7.5 \%)$ of 40 tumors with preserved or intermediate Fhit expression ( $\mathrm{p}=0.01$ ) (Fig. 1) (Table II).

We also analyzed the relationship between these results and the clinicopathological data (size, tumor location, macro- 
Table II. Fhit, Mlh1, Msh2 and P53 expression in early colorectal neoplasms.

\begin{tabular}{|c|c|c|c|c|c|c|c|c|c|c|c|}
\hline & & \multicolumn{2}{|c|}{ Fhit } & \multicolumn{2}{|c|}{ Mlh1 } & \multicolumn{3}{|c|}{ Msh2 } & \multicolumn{3}{|c|}{ P53 } \\
\hline & No. & $\begin{array}{l}\text { Preserved or } \\
\text { intermediate }\end{array}$ & $\begin{array}{c}\text { Reduced or } \\
\text { absent }\end{array}$ & + & - & & + & - & + & & - \\
\hline Adenoma & 30 & 30 & 0 & 29 & 1 & & 30 & 0 & 1 & $\bar{\square}$ & 29 \\
\hline CIA & 30 & 30 & $0 \square$ & 29 & 1 & 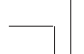 & 30 & 0 & 8 & & 22 \\
\hline EPC & 18 & 10 & $8=$ & 12 & 6 & $=$ & 17 & 1 & 7 & & 11 \\
\hline
\end{tabular}

Reduced or absent Fhit expression was markedly higher in EPCs than in adenomas and CIAs, respectively ( $\mathrm{p}=0.0001$ and 0.0001 ). Loss of Mlh1 expression was markedly higher in EPCs than in adenomas and CIAs, respectively ( $\mathrm{p}=0.008$ and 0.008 ). P53 overexpression was markedly higher in CIAs and EPCs than in adenomas, respectively ( $\mathrm{p}=0.02$ and 0.002 ).

Table III. Relationship between Fhit, Mlh1 Msh2, P53 protein expression and clinicopathological findings in early colorectal carcinomas.

\begin{tabular}{|c|c|c|c|c|c|c|c|c|c|}
\hline & \multirow[b]{2}{*}{ No. } & \multicolumn{2}{|c|}{ Fhit } & \multicolumn{2}{|c|}{ Mlh1 } & \multicolumn{2}{|c|}{ Msh2 } & \multicolumn{2}{|c|}{ P53 } \\
\hline & & $\begin{array}{l}\text { Preserved or } \\
\text { intermediate }\end{array}$ & $\begin{array}{l}\text { Reduced or } \\
\text { absent }\end{array}$ & + & - & + & - & + & - \\
\hline \multicolumn{10}{|c|}{ Depth of invasion } \\
\hline Mucosa & 31 & 30 & $1 \neg$ & 28 & 3 & 31 & 0 & 10 & 21 \\
\hline Submucosa & 17 & 10 & $7-$ & 13 & 4 & 16 & 1 & 5 & 12 \\
\hline \multicolumn{10}{|c|}{ Lymphatic invasion } \\
\hline Negative & 42 & 37 & 57 & 36 & 6 & 41 & 1 & 13 & 29 \\
\hline Positive & 6 & 3 & $3-$ & 5 & 1 & 6 & 0 & 2 & 4 \\
\hline \multicolumn{10}{|c|}{ Venous invasion } \\
\hline Negative & 44 & 39 & $5 \rightarrow$ & 38 & 6 & 43 & 1 & 14 & 30 \\
\hline Positive & 4 & 1 & $3-$ & 3 & 1 & 4 & 0 & 1 & 3 \\
\hline
\end{tabular}

Reduced or absent Fhit expression in early colorectal carcinomas was markedly higher in the cases of subumucosal invasion, lymphatic invasion and venous invasion, respectively ( $\mathrm{p}=0.001,0.0018$ and 0.0018$)$.

scopic feature, depth of invasion, and lymphatic and venous invasion). Reduced Fhit expression was detected in 1 (3\%) mucosal confined tumor and $7(41 \%)$ submucosal invading tumors among 48 early CRCs (30 CIAs and 18 EPCs). The incidence of reduced Fhit expression in the early CRCs was significantly higher in the submucosal invading tumors than mucosal confined tumors $(\mathrm{p}=0.001)$. Reduced Fhit expression was detected in $3(50 \%)$ of 6 cases involving lymphatic invasion and $3(75 \%)$ of 4 cases involving venous invasion. The incidence of reduced Fhit expression in early CRCs was significantly higher in the cases involving lymphatic invasion and venous invasion than in the cases with no lymphatic or venous invasion $(\mathrm{p}=0.0018)$. No significant associations were found among the Mlh1, Msh2 and P53 expression and other clinicopathological parameters (Table III).

HGM, MUC2 and CD10 expression in early colorectal neoplasms. HGM was more frequently expressed in adenomas
$(53 \%)$ and the adenomatous area of CIAs $(87 \%)$ than in carcinomatous area of CIAs $(37 \%)$ and EPCs $(22 \%)(\mathrm{p}<0.05)$. HGM expression in the carcinomatous components of CIAs tended to be decreased compared with that in the adenomatous components. MUC2 was more frequently expressed in adenomas $(97 \%)$ than in EPCs $(67 \%)(\mathrm{p}=0.008)$. Moreover, MUC2 was more frequently expressed in CIAs (83\%) than in EPCs $(67 \%)$, but the difference was not significant. CD10 was more frequently expressed in the carcinomatous area of CIAs $(33 \%)$ and EPCs $(39 \%)$ than in adenomas $(3 \%)(\mathrm{p}<0.05)$ (Fig. 2) (Table IV). There was no significant correlation between phenotypic expression and clinicopathological data in early colorectal neoplasms.

Relationship between phenotype and Fhit, Mlh1, Msh2 and P53 expression in early colorectal neoplasms. We analyzed that relationship between phenotype and Fhit, Mlh1, Msh2 and P53 expression in early colorectal neoplasms. In the 
A

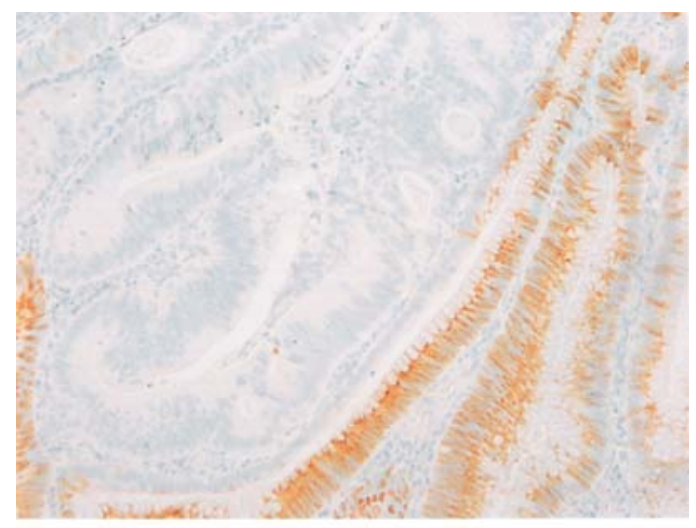

$\mathbf{B}$

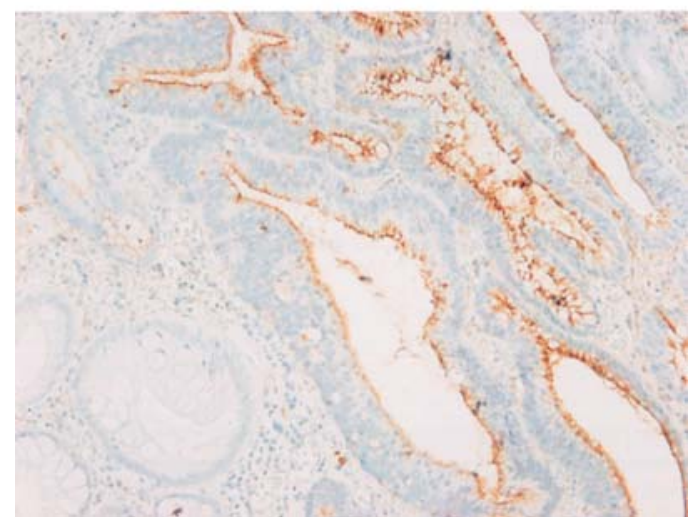

Figure 2. Phenotypic expression in normal and neoplastic tissue. (A) Positive MUC2 immunostaining of an adenomatous area of CIA and negative MUC2 immunostaining of a carcinomatous area of CIA. (B) Positive CD10 immunostaining of a carcinomatous area of CIA and negative CD10 immunostaining of a normal colonic epithelium.

Table IV. HGM, MUC2 and CD10 expression in early colorectal neoplasms.

\begin{tabular}{|c|c|c|c|c|}
\hline & No. & $\begin{array}{c}\text { HGM } \\
\text { Positive } \\
(\%)\end{array}$ & $\begin{array}{c}\text { MUC2 } \\
\text { Positive } \\
(\%)\end{array}$ & $\begin{array}{c}\text { CD10 } \\
\text { Positive } \\
(\%)\end{array}$ \\
\hline Adenoma & 30 & $16(53)-$ & $29(97)-$ & $1 \quad(3)=$ \\
\hline \multicolumn{5}{|l|}{ CIA } \\
\hline Adenomatous area & 30 & $26(87)$ & $30(100)$ & $0 \quad(0)$ \\
\hline Carcinomatous area & 30 & $11(37)-$ & $25(83)$ & $10(33)$ \\
\hline $\mathrm{EPC}$ & 18 & $4(22)-$ & $12(67)$ & $7(39)$ \\
\hline
\end{tabular}

HGM was more frequently expressed in adenomas and the adenomatous area of CIAs than in the cacrinomatous area of CIAs and EPCs, respectively ( $\mathrm{p}=0.04$ and 0.0001$)$. MUC2 was more frequently expressed in adenomas than in EPCs $(p=0.008)$. CD10 was more frequently expressed in the carcinomatous area of CIAs and EPCs than in adenomas, respectively ( $\mathrm{p}=0.006$ and 0.003$)$.

cases where CD10 expression was positive, 5 (50\%) of 10 CIAs and $4(57 \%)$ of 7 EPCs were positive for P53. While among the cases where CD10 expression was negative, 3
Table V. Relationship between CD10 and P53 expression in early colorectal carcinomas.

\begin{tabular}{lccc}
\hline & & \multicolumn{2}{c}{ CD10 } \\
\cline { 3 - 4 } & No. & Positive & Negative \\
\hline P53 & & & \\
Positive & 15 & 9 & 6 \\
Negative & 33 & 8 & 25 \\
\hline
\end{tabular}

The P53 overexpression rate was much higher in the CD10-positive cases than in the CD10-negative cases $(\mathrm{p}=0.04)$.

(15\%) of 20 CIAs and 3 (27\%) of 11 EPCs were P53 positive. The P53 overexpression rate was much higher in the CD10positive cases than CD10-negative cases $(\mathrm{p}=0.04)$ (Table V). There was no significant association between phenotype and Fhit, Mlh1, Msh2 expression.

\section{Discussion}

There are two opposing theories regarding the natural history of colorectal neoplasms, ACS and DN carcinogenesis $(1,2)$. $\mathrm{DN}$ cancer features a more rapid invasion than the ACS types of colorectal cancer, and in advanced cases, it is difficult to distinguish whether the cancer developed from DN or ACS. Moreover, DN carcinogenesis is thought to be characterized by superficial growth pattern (2-5). Therefore, studies on very early preneoplastic lesions are essential for understanding the molecular mechanism of colorectal carcinogenesis. In this study, we selected early colorectal neoplasms confined to the mucosa or submucosa, and assigned endoscopically resected CIAs and EPCs to ACS and DN carcinoma, respectively.

Alterations and abnormal transcripts of the FHIT gene have been reported in a number of primary human tumors, including CRCs (6-9). Recent studies have revealed that reduced Fhit expression is associated with a loss of MMR protein expression in advanced CRCs (11-13). The results of several studies have indicated that the immunohistochemical analysis of Fhit, Mlh1, and Msh2 could be a reliable and rapid screening procedure for the identification of these gene alterations (29-33). In this study, abnormal Fhit and Mlh1 expression was observed in 44.4 and $33.3 \%$ of the EPCs respectively, but rarely in adenomas and CIAs. In addition, our data confirmed an association between Fhit and Mlh1 expression. Kuwai et al (9) found the frequency of reduced Fhit expression to be significantly higher in the depressed type than polypoid type. In our study, 12 of 18 EPCs were flat or depressed. These results provide support for the hypothesis that the molecular pathogenesis of DN carcinogenesis is distinct from that of ACS with respect to Fhit and Mlh1 expression. Moreover, in a previous study, we showed that the frequency of loss of Fhit and Mlh1 expression was significantly higher in poorly differentiated specimens of advanced CRCs than in well/moderately differentiated specimens (11). Taken together, these findings suggest that 
some proportion of poor cases may originate from EPCs, and that the carcinogenesis of poorly differentiated CRCs may depend to some degree on a DN pathway. We also found that submucosal and lymphatic or venous invasive tumors exhibited reduced Fhit expression more frequently than the others. Previous reports found that reduced expression of Fhit is associated with progression of CRCs, suggesting that reduced Fhit expression may predict the progression of EPCs $(8,9)$.

$p 53$ is a tumor suppressor gene located on $17 q 13$ and its mutations are thought to be involved in the adenoma to carcinoma conversion in colorectal carcinogenesis $(34,35)$. Previous reports indicated that $p 53$ is related to the enlargement and deeper invasion of superficial-type early CRC, regardless of the sequence of development (36). The present study found that the P53-positive rate was much higher in CIAs and EPCs than in adenomas. However, we could not find a relationship between P53 and submucosal invasion. This discrepancy might be related to differences in the selection of early colorectal neoplasms.

CD10 is present on the luminal surfaces of small intestinal absorptive cells, and rarely in the large intestine (23) and overexpression of CD10 may be associated with the development and progression of CRCs (25), as well as an increased risk of liver metastasis (37). The present findings clearly demonstrate a significant increase of CD10 expression from adenomas to CIAs and EPCs. The present study also found that 9 of 17 CD10-positive cases and 6 of 31 CD10-negative cases were P53 positive. The P53-positive rate was much higher in the CD10-positive cases than in the CD10-negative cases. These findings are consistent with previous reports and suggest that CD10-positive carcinomas are aggressive, and overexpression of CD10 seems to be related to the development of colorectal neoplasms (25).

MUC2 is a $550-\mathrm{kDa}$ glycoprotein that is regarded as the principal secretory mucin in the colorectum and is specific for goblet cells. It has been reported that MUC2 is abundantly expressed in normal colonic goblet cells and that expression of MUC2 mucin is decreased in colonic adenoma and carcinoma cells compared with normal mucosa $(21,22,32,38,39)$. Velcich et al (40) reported that MUC2 is involved in the suppression of CRC in mice. Some studies have indicated that reduced MUC2 expression might be related to the malignant transformation of colorectal adenoma and carcinoma $(25,39,41)$. In our study, MUC2 mucin expression was significantly lower in the CIAs than in the adenomas. Therefore, the present study confirmed that the expression of MUC2 mucin is associated with malignant transformation particularly in the early stages of ACS. Moreover, MUC2 expression was lower in the EPCs than in the CIAs, but the difference was not significant, which is consistent with the findings of some investigators $(25,39,42)$. Our data suggest that the reduction of MUC2 expression may be associated with the occurrence and progression of CRCs in both the ACS pathway and DN carcinogenesis.

HGM, which is identical to MUC5AC (43), is expressed by mucous cells on the surface of the gastric epithelium and by goblet cells of the fetal and precancerous colon, but not by those of the normal colon (44). Previous studies have reported that MUC5AC is not expressed in the normal colonic mucosa, but shows increased expression in colorectal neoplasms (45).
In the present study, markedly strong expression of HGM was observed not only in adenomas, but also in the adenomatous component of CIAs. In contrast, HGM was less frequently expressed in the carcinomatous component of CIAs and EPCs. Further studies are needed to clarify the association between gastric phenotypic expression and the development of colorectal neoplasms. Thus, there were significant differences between the phenotypic expression of the adenomatous area and adenocarcinomatous area in early colorectal neoplasms, suggesting that an analysis of phenotypic expression may have potential in the prediction of malignant transformation.

In conclusion, reduced or absent expression of Fhit and Mlh1 may be associated with the development and progression of DN carcinoma. Moreover, our results suggest that phenotypic change and P53 expression contribute to the malignant transformation of colorectal precursor lesions, at least in the Japanese population examined by us. Further characterization and elucidation of DN carcinogenesis may be expected to yield important new insights into the understanding and management of CRCs.

\section{Acknowledgements}

We thank Goshi Shiota (Division of Molecular and Genetic Medicine, Faculty of Medicine, Tottori University) and Hisao Ito (Division of Organ Pathology, Faculty of Medicine, Tottori University) for helpful suggestions.

\section{References}

1. Vogelstein B, Fearon ER, Hamilton SR, et al: Genetic alterations during colorectal-tumor development. N Engl J Med 319: 525-532, 1998

2. Kuramoto $\mathrm{S}$ and Oohara T: Minute cancers arising de novo in the human large intestine. Cancer 61: 829-834, 1988.

3. Muto T, Kamiya J, Sawada T, et al: Small 'flat adenoma' of the large bowel with special reference to its clinicopathologic features. Dis Colon Rectum 28: 847-851, 1985.

4. Shimoda T, Ikegami M, Fujisaki J, Matsui T, Aizawa S and Ishikawa E: Early colorectal carcinoma with special reference to its development de novo. Cancer 64: 1138-1146, 1989.

5. Kudo S, Kashida H, Nakajima T, Tamura $S$ and Nakajo K: Endoscopic diagnosis and treatment of early colorectal cancer. World J Surg 21: 694-701, 1997.

6. Huebner K and Croce CM: cancer and the FRA3B/FHIT fragile locus: it's a HIT. Br J Cancer 88: 1501-1506, 2003.

7. Ohta M, Inoue H, Cotticelli MG, et al: The FHIT gene, spanning the chromosome 3 p 14.2 fragile site and renal carcinomaassociated $\mathrm{t}(3 ; 8)$ breakpoint, is abnormal in digestive tract cancers. Cell 84: 587-597, 1996.

8. Hao XP, Willis JE, Pretlow TG, Rao JS, MacLennan GT, Talbot IC and Pretlow TP: Loss of fragile histidine triad expression in colorectal carcinomas and premalignant lesions. Cancer Res 60: 18-21, 2000.

9. Kuwai T, Tanaka S, Kaio E, et al: Clinical significance of Fhit expression in development of colorectal carcinoma of various macroscopic types. Int J Mol Med 12: 437-442, 2003.

10. Mimori K, Ishii H, Nagahara H, et al: FHIT is up-regulated by inflammatory stimuli and inhibits prostaglandin E2-mediated cancer progression. Cancer Res 66: 2683-2690, 2006.

11. Andachi H, Yashima K, Koda M, et al: Reduced Fhit expression is associated with mismatch repair deficiency in human advanced colorectal carcinoma. Br J Cancer 87: 441-445, 2002.

12. Mori M, Mimori K, Masuda T, Yoshinaga K, Yamashita K, Matsuyama $\mathrm{A}$ and Inoue $\mathrm{H}$ : Absence of $\mathrm{Msh} 2$ protein expression is associated with alteration in the FHIT locus and Fhit protein expression in colorectal carcinoma. Cancer Res 61: 7379-7382, 2001 . 
13. Sarli L, Bottarelli L, Azzoni C, et al: Abnormal Fhit protein expression and high frequency of microsatellite instability in sporadic colorectal cancer. Eur J Cancer 40: 1581-1588, 2004.

14. Koseki K, Takizawa T, Koike M, Ito M, Nihei Z and Sugihara K: Distinction of differentiated type early gastric carcinoma with gastric type mucin expression. Cancer 89: 732-742, 2000

15. Tajima Y, Shimoda T, Nakanishi Y, et al: Gastric and intestinal phenotypic marker expression in gastric carcinomas and its prognostic significance: immunohistochemical analysis of 136 lesions. Oncology 61: 212-220, 2001.

16. Kabashima A, Yao T, Sugimachi $\mathrm{K}$ and Tsuneyoshi M: Relationship between biologic behavior and phenotypic expression in intramucosal gastric carcinomas. Hum Pathol 33: 80-86, 2002.

17. Kawaguchi K, Yashima K, Koda M, et al: Fhit expression in human gastric adenomas and intramucosal carcinomas: correlation with Mlh1 expression and gastric phenotype. Br J Cancer 90: 672-677, 2004.

18. Reis CA, David L, Correa P, et al: Intestinal metaplasia of human stomach displays distinct patterns of mucin (MUC1, MUC2, MUC5AC, and MUC6) expression. Cancer Res 59: 1003-1007, 1999.

19. Tatematsu M, Tsukamoto T and Inada K: Stem cells and gastric cancer: role of gastric and intestinal mixed intestinal metaplasia. Cancer Sci 94: 135-141, 2003.

20. Tsukamoto T, Inada K, Tanaka H, et al: Down-regulation of a gastric transcription factor, Sox2, and ectopic expression of intestinal homeobox genes, $\mathrm{Cdx} 1$ and $\mathrm{Cdx} 2$ : inverse correlation during progression from gastric/intestinal-mixed to complete intestinal metaplasia. J Cancer Res Clin Oncol 130: 135-145, 2004.

21. Ajioka Y, Watanabe H and Jass JR: MUC1 and MUC2 mucins in flat and polypoid colorectal adenomas. J Clin Pathol 50: 417-421, 1997.

22. Ajioka Y, Allison LJ and Jass JR: Significance of MUC1 and MUC2 mucin expression in colorectal cancer. J Clin Pathol 49: 560-564, 1996

23. Yao T, Tsutsumi S, Akaiwa Y, Takata M, Nishiyama K, Kabashima A and Tsuneyoshi M: Phenotypic expression of colorectal adenocarcinomas with reference to tumor development and biological behavior. Jpn J Cancer Res 92: 755-761, 2001

24. Takata M, Yao T, Nishiyama KI, Nawata H and Tsuneyoshi M: Phenotypic alteration in malignant transformation of colonic villous tumours: with special reference to a comparison with tubular tumours. Histopathology 43: 332-339, 2003.

25. Iwase T, Kushima R, Mukaisho K, Mitsuhuzi S, Okanoue T and Hattori T: Overexpression of CD10 and reduced MUC2 expression correlate with the development and progression of colorectal neoplasms. Pathol Res Pract 201: 83-91, 2005.

26. Japanese General Rules for Clinical and Pathological Studies on Cancer of the Colon, Rectum, and Anus. Japanese Society for Cancer of the Colon and Rectum, 1998.

27. Saitoh Y, Waxman I, West AB, et al: Prevalence and distinctive biologic features of flat colorectal adenomas in a North American polulation. Gastroenterology 120: 1657-1665, 2001.

28. Beak MJ, Kang H, Kim SE, Park JH, Lee JS, Paik YK and Kim H: Expression of hMLH1 is inactivated in the gastric adenomas with enhanced microsatellite instability. $\mathrm{Br} \mathrm{J}$ Cancer 85: 1147-1152, 2001
29. Thibodeau SN, French AJ, Roche PC, et al: Altered expression of hMSH2 and hMLH1 in tumors with microsatellite instability and genetic alterations in mismatch repair genes. Cancer Res 56: 4836-4840, 1996

30. Sozzi G, Pastorino U, Moiraghi L, et al: Loss of FHIT function in lung cancer and preinvasive bronchial lesions. Cancer Res 58: 5032-5037, 1998.

31. Marcus VA, Madlensky L, Gryfe R, et al: Immunohistochemistry for hMLH1 and hMSH2: a practical test for DNA mismatch repair-deficient. Am J Surg Pathol 23: 1248-1255, 1999.

32. Birrer MJ, Hendricks D, Farley J, Sundborg MJ, Bonome T, Walts MJ and Geradts J: Abnormal Fhit expression in malignant and premalignant lesions of the cervix. Cancer Res 59: 5270$5274,1999$.

33. Mori M, Mimori K, Shiraishi T, et al: Altered expression of Fhit in carcinoma and precarcinomatous lesions of the esophagus. Cancer Res 60: 1177-1182, 2000.

34. Baker SJ, Preisinger AC, Jessup JM, et al: p53 gene mutations occur in combination with $17 \mathrm{p}$ allelic delections as late events in colorectal tumorigenesis. Cancer Res 50: 7717-7722, 1990.

35. Ilyas M, Straub J, Tomlinson IPM and Bodmer WF: Genetic pathways in colorectal and other cancers. Eur J Cancer 35 1986-2002, 1999.

36. Wasa R, Matsukuma S, Abe H, Kuwabara N, Suda K, Arakawwa A and Kitamura S: Histopathological studies of superficial-type early colorectal carcinoma. Cancer 77: 44-50, 1996.

37. Fujimoto Y, Nakanishi Y, Sekine S, Yoshimura K, Akasu T, Moriya Y and Shimoda T: CD10 expression in colorectal carcinoma correlates with liver metastasis. Dis Colon Rectum 48: 1883-1889, 2005.

38. Buisine MP, Janin A, Maunoury V, et al: Aberrant expression of a human mucin gene (MUC5AC) in rectosigmoid villous adenoma. Gastroenterology 110: 84-91, 1996.

39. Mizoshita T, Tsukamoto T, Inada KI, et al: Loss of MUC2 expression correlates with progression along the adenomacarcinoma sequence pathway as well as de novo carcinogenesi in the colon. Histol Histopathol 22: 251-260, 2007.

40. Velcich A, Yang W, Heyer J, et al: Colorectal cancer in mice genetically deficient in the mucin Muc2. Science 295: 1726-1729, 2002.

41. Li A, Goto M, Horinouchi M, et al: Expression of MUC1 and MUC2 mucins and relationship with cell proliferative activity in human colorectal neoplasia. Pathol Int 51: 853-860, 2001.

42. Blank M, Klussmann E, Krasagakes SK, et al: Expression of MUC2-mucin in colorectal adenomas and carcinomas of different histological types. Int J Cancer 59: 301-306, 1994.

43. Bara J, Gautier R, Daher N, Zaghouani H and Decaens C: Monoclonal antibodies against oncofetal mucin M1 antigens associated with precancerous colonic mucosae. Cancer Res 46: 3983-3989, 1986.

44. Bara J, Gautier R, Mouradian P, Decaens C and Daher N Oncofetal mucin M1 epitope family: characterization and expression during colonic carcinogenesis. Int J Cancer 47: 304-310, 1991

45. Bartman AE, Sanderson SJ, Ewing SL, Niehans GA, Wiehr CL, Evans MK and Ho SB: Aberrant expression of MUC5AC and MUC6 gastric mucin genes in colorectal polyps. Int J Cancer 80: 210-218, 1999. 